\title{
Evaluation of Bacillus - Calmette- Guerin (BCG) Immunogenicity in Indigenous Calves under Field Condition
}

\author{
M. Asokkumar ${ }^{\text {** }}$, G. Selvaraju' ${ }^{1}$, S. Manoharan², K.G. Tirumurugaan³, \\ V. Maroudam ${ }^{4}$ and M. Vijayabharathi ${ }^{1}$ \\ ${ }^{1}$ Department of Veterinary Preventive Medicine, Madras Veterinary College, TANUVAS, \\ Chennai-7, Tamil Nadu, India \\ ${ }^{2}$ Vaccine Research Centre - Bacterial Vaccines, TANUVAS, Chennai-51, Tamil Nadu, India \\ ${ }^{3}$ Department of Animal Biotechnology, Madras Veterinary College, TANUVAS, Chennai-7, \\ Tamil Nadu, India \\ ${ }^{4}$ (Penn. State University, USA and Melinda Gates Foundation), Animal TB control Project, \\ TANUVAS, Chennai-600 051, Tamil Nadu, India \\ *Corresponding author
}

\section{A B S T R A C T}

Keywords

Bovine

Tuberculosis- BCG

- Immune Response

- Indigenous Calves

Article Info

Accepted:

15 September 2018

Available Online:

10 October 2018
Bovine tuberculosis (bTB) remains a major problem causing huge economic loss in livestock farming. It can spread to humans from infected cattle either through aerosol or by consumption of contaminated dairy products to cause zoonotic tuberculosis. Current Indian diagnostic approach is by intra dermal injection of PPD (tuberculin skin test) derived from the Mycobacterium bovis (bovine PPD) wherein the infected animals provokes a measurable skin reaction. BCG vaccination is not used to control bovine tuberculosis because of minimal data available on its vaccine immunogenicity and efficacy studies in cattle. Thus it's important to test the BCG vaccine performance in the Indian cattle population. The present study aimed at evaluating the BCG vaccine immunogenicity testing in young sahiwal calves. The bTB screening and vaccine immunogenicity were evaluated using the OIE approved Interferon gamma release assay (IGRA) using BOVIGAM ${ }^{\mathrm{TM}}$ kit. BCG was titrated with $10^{5}$ colony forming units (CFU) and observed from the present study that low dose of $10^{5}(\mathrm{CFU})$ is found to be effective for inducing significant bTB specific protective IFN-gamma at day 14 post vaccination. Thus the conventional BCG vaccination shall be considered as an approach for the control as well as reducing the bio burden of bovine tuberculosis along with complementary diagnosis.

\section{Introduction}

Mycobacterium bovis, the causative agent of bovine tuberculosis infects a wide range of hosts, including domestic livestock, wildlife and humans and is a major economic and public health problem in numerous countries. Although regular tuberculin testing and removal of infected animals has been successful in eradicating or markedly reducing bovine tuberculosis from cattle herds in industrialized countries, these control measures are not affordable or acceptable in 
many parts of the world especially in developing and under developed nations. More than $94 \%$ of the world's population lives in countries in which the control of bovine tuberculosis in cattle or buffaloes is limited or absent (Cousins, 2001) and a cost effective control strategy such as immunization would be extremely valuable for these countries in view of reducing its bioburden among animals and thereby human as well. Immunization would also be applicable in countries which have a sylvatic reservoir of infection where conventional control strategies such as testing and slaughter of reactors are less effective. In India, the current diagnostic approach is by tuberculin skin test (PPD) in which a crude denatured antigen from the Mycobacterium bovis (bovine PPD) is used, where the infected animals provokes a measurable skin reaction. BCG immunization is not practiced to control bovine tuberculosis because of minimal data available on its vaccine immunogenicity and efficacy studies in cattle. By considering these facts, a study was conducted in indigenous calves to evaluate the immune response against BCG immunization under field conditions.

\section{Materials and Methods}

\section{Experimental animals}

Forty numbers of Sahiwal calves, aged between 6 to 12 weeks from a well maintained private farm (Farm Kadalur) was used for this study and were screened with Bovigam kit before starting immunization trial to rule out positive cases.

\section{Vaccine}

Live, attenuated bacterial strain (Danish 1331) of Mycobacterium bovis, Bacillus CalmetteGuerin (BCG) was used for active immunization against tuberculosis.

Experimental design and immunization
The experiment was designed in such a way that twenty naïve calves were randomly allocated into two groups as controlled (unvaccinated) and BCG vaccinated in the same farm. The vaccinated groups were inoculated subcutaneously with $10^{5}$ cfu in 2 $\mathrm{ml}$ diluent on the neck and the heparinized blood samples were collected from all calves at day $0,14,30,60$ and 90 post vaccination.

\section{Mycobacterial proteins}

Mycobacterial proteins such as ESAT-6 and CFP-10 (Translational Research Platform for Veterinary Biologicals, TANUVAS), Bovine and Avian tuberculin PPD (Prionics AG, Switzerland) and Concanavalin A were used for stimulation of PBMC from heparinized blood to perform interferon gamma release assay (IGRA).

\section{Blood collection and IFN- $\gamma$ assay}

The blood samples collected from naïve and immunized calves in heparinized sterile vacuum containers were processed within 6 hrs after collection for PBMC stimulation with different mycobacterial proteins. The culture supernatants of mycobacterial proteins stimulated blood were harvested after incubation at $37 \pm 0.5^{0} \mathrm{C}$ for $24 \mathrm{hrs}$ with $5 \%$ $\mathrm{CO}_{2}$ incubator (Lark, India).

The 96 well cell culture plate (Corning, USA) was coated with immune capture antibody $(100 \mu \mathrm{l} /$ well $)$ at a concentration of $1: 2000$ dilution and incubated over night at $5 \pm 3^{0} \mathrm{C}$. All steps were carried out in the room temperature for incubation with shaking at 500 - $600 \mathrm{rpm}$. For each step, washing was done with PBST for 3 times and then allowed to dry by tapping. Blocking was performed with $2 \%$ skimmed milk powder (Difco, BD) at $200 \mu \mathrm{l} /$ well and incubated at RT for $1 \mathrm{hr}$. Antigen and standards (10000 to $4 \mathrm{pg} / \mathrm{ml}$ ) 
were prepared and each well was added with $100 \mu \mathrm{l}$ and then incubated for $1 \mathrm{hr}$. After that, Biotynalated (Bio-Rad, UK) antibody (1:4000,) and streptavidin (Bio-Rad, UK) conjugate $(1: 4000)$ were added at $100 \mu \mathrm{l} /$ well and incubated for $1 \mathrm{hr}$ and 30 minutes respectively. The TMB substrate was prepared as per standard procedure and incubated for 30 min. after wells were added with $100 \mu \mathrm{l}$. Finally the reaction was stopped with $0.18 \mathrm{M}$ $\mathrm{H}_{2} \mathrm{SO}_{4}(100 \mu \mathrm{l} /$ well $)$ and the result was read at $450 \mathrm{~nm}$ using ELISA plate reader (Bio-Rad, UK). Interpretation was executed through a standard curve plotted with the absorbance values against the respective standard concentrations. The concentration of test sample is extrapolated from the standard graph.

\section{Results and Discussion}

A total of forty sahiwal calves aged between 6 to 12 weeks were screened for tuberculosis (bTB) with BOVIGAM ${ }^{\circledR}$ Gamma Interferon Assay and the levels of Interferon Gamma (IFN gamma) response to Avian PPD, Bovine PPD and ESAT6:CFP10 were determined. The stimulation index value of less than 0.1 was considered as naïve population for this study with only having the basal level of interferon gamma.

The immunogenicity was estimated by amount of INF- $\gamma$ release as a surrogate of vaccination efficiency from whole blood stimulated with mycobacterial proteins like bovine PPD, avian PPD and ESAT-6:CFP 10 antigens in an IFN$\gamma$ release assay for both vaccinated and control groups. The blood stimulated with no antigens showed value of $<0.1$, which indicated the basal level of IFN- $\gamma$ without stimulation. But the OD values of bovine and avian PPD were high compared to nil antigen stimulation. The OD values to highly defined fusion proteins (ESAT-6+CFP 10) remains low as the nil antigens stimulation which can be an indicator of the actual disease status of the population. This strategy enabled us to select the naïve populations for this immunogenicity study (Fig. 1). This results were in compliance with BCG vaccination of neonatal calves induced a higher level of immunity against bovine tuberculosis than that seen in calves vaccinated at 5-6 months of age (Buddle et al., 2003b, c; Hope et al., 2005 and 2011).

The groups vaccinated with BCG subcutaneously showed the INF- $\gamma$ peaked at day 14 post vaccination and then reduced by day 30 but vaccinated with higher value than pre-vaccination level (Fig. 2), whereas the unvaccinated group vaccinated relatively with constant titre with minor fluctuations which might be due to environmental mycobacterial exposure (Fig. 3). There was no significant difference observed between these groups at day 0 , since they had been screened and naïve calves were selected for this study. But the concentration of INF- $\gamma$ reached peak at day 14 post vaccinations and higher protective value were found in the vaccinated group than in the control group. The difference between vaccinated and control groups were statistically significant $(\mathrm{P}<0.05)$ until day 90 post vaccination. Similar findings were reported by Buddle et al., (2002 and 2011) and Ameni et al., (2010) where the mean responses to bovine PPD for both cytokines were greater for vaccinated groups than unvaccinated groups $(\mathrm{P}<0.01)$ at 2,4 and 7 weeks after vaccination and also noted markedly reduced response by 5 weeks post vaccination. They have also stated that significant protection against the development of tuberculous was conferred on calves by sub cutaneous vaccination with $6 \times 10^{4}$ or $6 \times 10^{6}$ cfu of BCG. In contrast, Wedlock et al., (2005 and 2007) noticed that the group vaccinated with BCG produced strong INF- $\gamma$ responses to bovine PPD by 3 weeks and $8-13$ weeks after initial vaccination. 


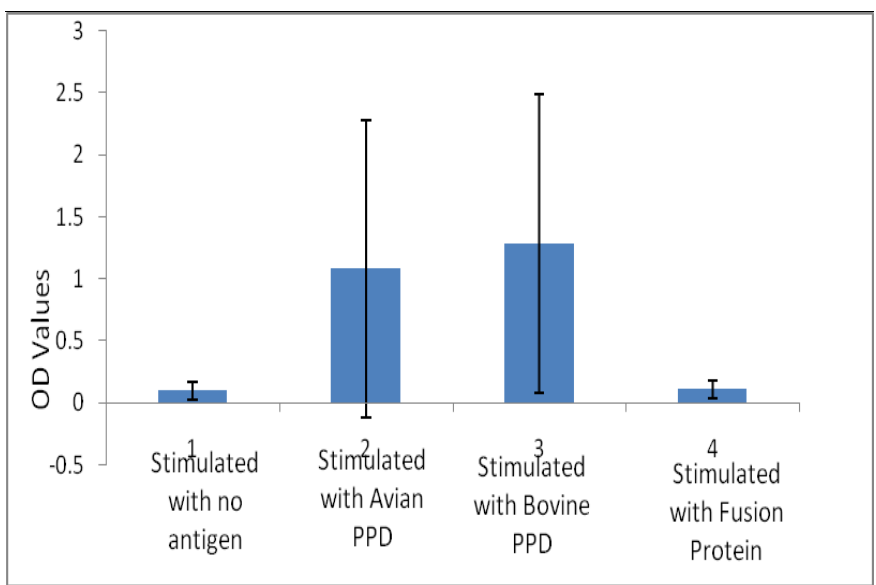

Fig.1 IGRA- Optical Density (OD) values (450 nm)

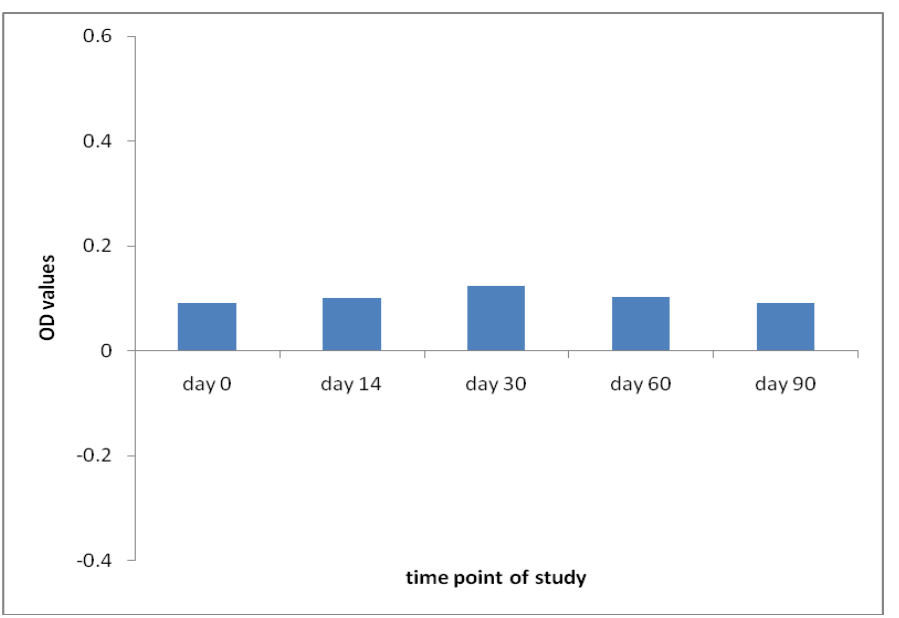

Fig.3 IFN gamma response of un-vaccinated control animals

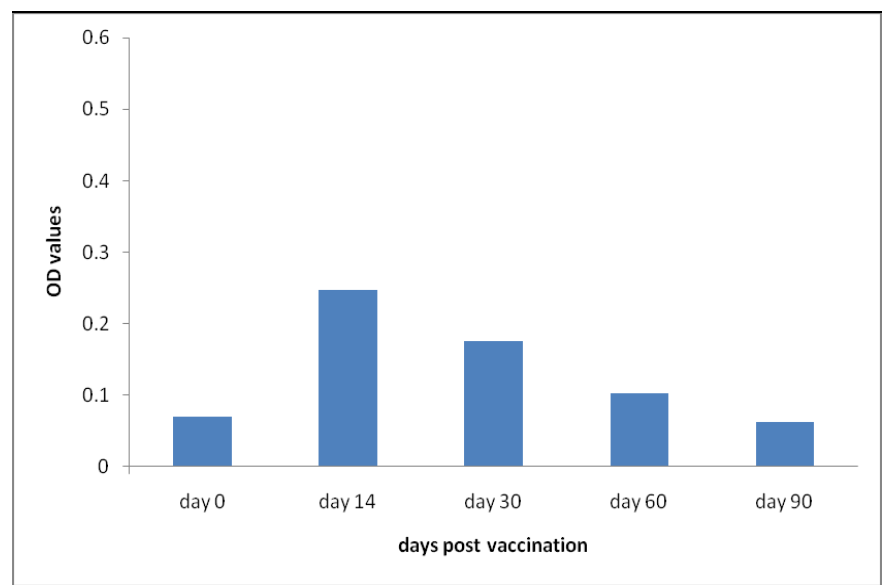

Fig.2 IFN gamma response in BCG vaccinated calves

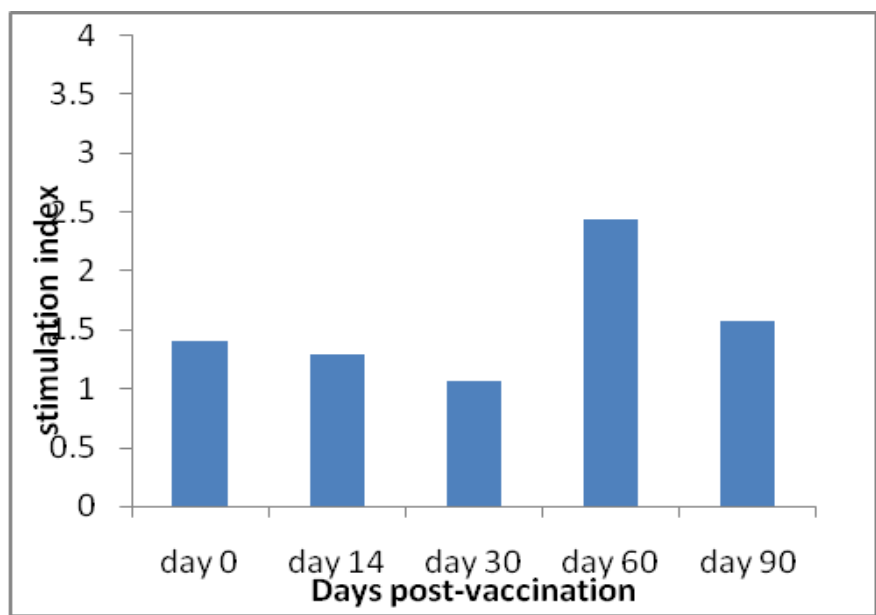

Fig.4 Stimulation index of ESAT6:CFP10 in BCG vaccinated animals

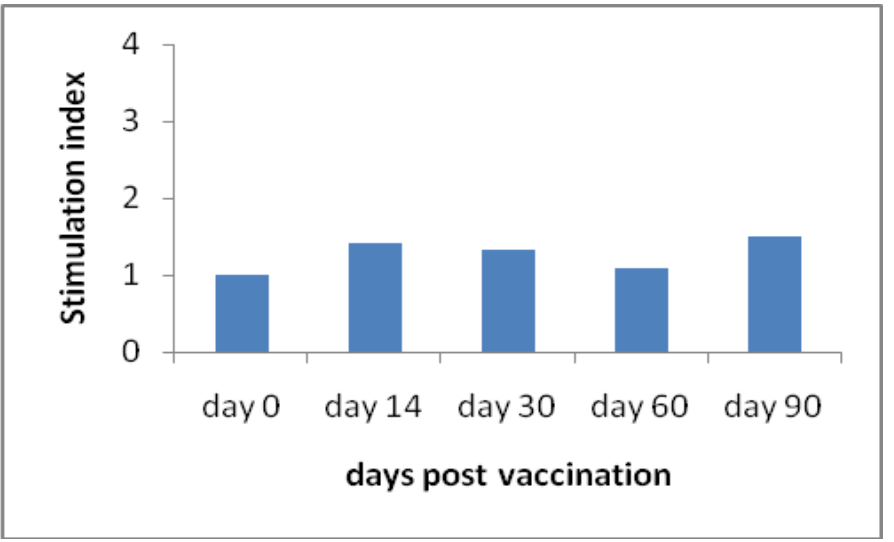

Fig.5 Stimulation index of ESAT6:CFP10 in vaccinated animals

The stimulation index of fusion proteins ESAT-6+CFP10 were performed in vaccinated as well as control groups during the study (day 0, 14, 30, 60 and 90 post vaccination) and found no much variation in the stimulation index value of vaccinated and control animals and the value remained below the cutoff value of 3 throughout the study 
period (Fig. 4 and 5). Unfortunately we could not able to execute challenge study due to ethical issues. Whelan et al., (2010) reported the defined protein cocktail did not induce a response in any of BCG vaccinated calves whereas the protein cocktail elicited delayedtype hypersensitivity (DTH) skin test responses in $78 \%$ of naturally infected tuberculin-positive cattle. All vaccinated animals when injected intra-dermally with ESAT6-CFP10 fusion protein showed no DTH response. The difference in skin thickness for all animals was below 0.5. Hence BCG vaccination doesn't cross-react with skin testing using TB specific ESAT6CFP10 fusion protein.

The study revealed that the BCG vaccine has the potential to reduce the bioburden of tuberculosis in calves by reducing the bacterial load and may be employed as calf hood immunization in view of protecting primary Ghon focus infection at young age as practiced in human beings. BCG vaccination of neonatal calves reduces the level of bacterial colonization and also severity of disease following $M$. bovis infection (Thom et. al., 2012). Vaccination against bovine tuberculosis is warranted to become an important disease control strategy in developing countries like India, which cannot afford a test and slaughter control programme, or in countries which have a sylvatic reservoir of Mycobacterium bovis infection.

\section{Acknowledgements}

This study was carried out as part of Ph.D thesis in Veterinary Preventive Medicine to be submitted to the Tamil Nadu Veterinary and Animal Sciences University (TANUVAS) by the first author. The authors are grateful to the farm Kadalur, Kanchipuram District, Tamil Nadu, Dr. G. Dhinakar Raj, Director, Translational Platform for Veterinary Biologicals and Dr.
P.I. Ganesan, then Director of Centre for Animal Health Studies, TANUVAS for providing the facility to carry out this work.

\section{References}

Ameni G, M Vordermeier, A Aseffa, DB Young and Hewinson RG. 2010. Field evaluation of the efficacy of Mycobacterium bovis bacillus CalmetteGuerin against bovine tuberculosis in neonatal calves in Ethiopia. Clin. Vaccine Immunol. 17:1533-1538.

Buddle B M, B J Wards, F E Aldwell, D M Collins and De Lisle, GW. 2002. Influence of sensitisation to environmental mycobacteria on subsequent vaccination against bovine tuberculosis. Vaccine, 20:1126-1133.

Buddle, B. M., D.N. Wedlock, M. Denis, H. M. Vordermeier, and Hewinson R.G. 2011. Update on vaccination of cattle and wildlife populations against tuberculosis. Veterinary Microbiology. 151: 14-22.

Buddle, B.M., D.N Wedlock, N.A. Parlane, L.A. Corner, G.W. De Lisle and Skinner, M.A., 2003c. Revaccination of neonatal calves with Mycobacterium bovis BCG reduces the level of protection against bovine tuberculosis induced by a single vaccination. Infect. Immun. 71, 6411-6419.

Buddle, B.M., J.M Pollock, M.A. Skinner and Wedlock, D.N., 2003b.Development of vaccines to control bovine tuberculosis in cattle and relationship to vaccine development for other intracellular pathogens. Int. J. Parasitol. 33, 555566.

Cousins D V, 2001. Mycobacterium bovis infection and control in domestic livestock. Rev Sci Tech., 20(1):71-85.

Hope JC, ML Thom, M McAulay, E Mead, HM Vordermeier, D Clifford, RG Hewinson and Villarreal-Ramos B. 
2011. Identification of surrogates and correlates of protection in protective immunity against Mycobacterium bovis infection induced in neonatal calves by vaccination with $M$. bovis BCG Pasteur and $M$. bovis BCG Danish. Clin. Vaccine Immunol. 18:373-379.

Hope, J.C., M.L. Thom, B Villarreal-Ramos, H.M. Vordermeier, R.G. Hewinson and Howard, C.J., 2005. Vaccination of neonatal calves with Mycobacterium bovis $\mathrm{BCG}$ induces protection against intranasal challenge with virulent $M$. bovis. Clin. Exp. Immunol. 139: 48-56.

Thom, M. L., M. McAulay, H. M. Vordermeier, D. Clifford, R. G. Hewinson, B. Villarreal-Ramos and J. C. Hope. 2012. Duration of Immunity against Mycobacterium bovis following Neonatal Vaccination with Bacillus Calmette-Guérin, Danish: Significant
Protection against Infection at 12, but Not 24, Months. Clin. Vaccine Immunol. 19(8):1254.

Wedlock DN, Denis M, Vordermeier HM, Hewinson RG, Buddle BM. 2007. Vaccination of cattle with Danish and Pasteur strains of Mycobacterium bovis BCG induce different levels of IFN post-vaccination, but induce similar levels of protection against bovine tuberculosis. Vet. Immunol. Immunopathol. 118:50 -58.

Wedlock, D.N., B.Vesosky, M.A. Skinner, G.W. de Lisle, I.M. Orme andB.M. Buddle. 2000. Vaccination of cattle with Mycobacterium bovis culture filtrate proteins and interleukin-2 for protection against bovine tuberculosis. Infect. Immun. 68: 5809-5815.

\section{How to cite this article:}

Asokkumar, M., G. Selvaraju, S. Manoharan, K.G. Tirumurugaan, V. Maroudam and Vijayabharathi, M. 2018. Evaluation of Bacillus - Calmette- Guerin (BCG) Immunogenicity in Indigenous Calves under Field Condition. Int.J.Curr.Microbiol.App.Sci. 7(10): 1939-1944. doi: https://doi.org/10.20546/ijcmas.2018.710.223 